\title{
Instantáneas
}

\section{Estrategia novedosa para reducir radicalmente la mortalidad por causas cardiovasculares}

Los problemas cardiovasculares prevenibles, tales como el infarto y la embolia cerebral, figuran entre las causas más importantes de muerte en las sociedades industrializadas. Para prevenirlos es necesario lograr cambios en la dieta y en el estilo de vida de la población que a todas luces son difíciles de conseguir y que podrían demorar varias generaciones en producirse. En el entretiempo, el uso de fármacos es el único recurso a nuestro alcance para reducir los factores de riesgo establecidos: hipertensión arterial, hipercolesterolemia, aumento de la actividad plaquetaria, elevación de las concentraciones séricas de lipoproteínas de baja densidad (LBD) y, posiblemente, de las concentraciones séricas de homocisteína. Hasta ahora, sin embargo, el control de estos factores no se ha acompañado de una reducción importante de la incidencia de trastornos cardiovasculares debido principalmente a que los fármacos suelen prescribirse para el tratamiento de problemas aislados en personas con valores clínicos en el extremo superior de la distribución poblacional, en quienes se busca reducir dichos valores al promedio observado en la población. Para que una estrategia preventiva surta los efectos deseados, es preciso dirigirla a todas las personas que se encuentran en riesgo, independientemente de la magnitud de este, y combatir los diversos factores de riesgo en conjunto, procurando reducirlos todo lo posible. Guiados por estos conceptos, investigadores en el Reino Unido han ideado una estrategia terapéutica a base de una píldora con seis componentes para la reducción simultánea de todos los factores de riesgo citados. La eficacia de la píldora fue confirmada mediante un estudio en que se examinaron su efecto sobre la incidencia de episodios clínicos de isquemia coronaria y de accidentes cerebrovasculares, además de la frecuencia de efectos adversos y el número de años de vida ganados. Los resultados permitieron concluir que si todas las personas con problemas cardiovasculares, más todas las que pasan de determinada edad (p. ej., los 55 años), tomaran este tipo de píldora a diario, la incidencia de accidentes cerebrovasculares y de episodios de isquemia coronaria se reduciría en más de $80 \%$. (Wald NJ,
Law MR. A strategy to reduce cardiovascular disease by more than $80 \%$. BMJ 2003;326:1419-1425.) 